\title{
Substâncias ergásticas foliares de espécies amazônicas de Oenocarpus Mart. (Arecaceae): caracterização histoquímica e ultra-estrutural ${ }^{1}$
}

\author{
Rolf Junior Ferreira SILVA ${ }^{*}$, Raimunda Conceição de Vilhena POTIGUARA ${ }^{3}$ \\ RESUMO \\ O presente estudo teve por objetivo diagnosticar e caracterizar as substâncias ergásticas foliares de Oenocarpus bacaba Mart., \\ O. distichus Mart., O. mapora H. Karst. e O. minor Mart. através de microscopias óptica e eletrônica de varredura, análises \\ histoquímicas e microanálises físicas. Secçôes transversais e longitudinais, assim como maceração foram realizadas em material \\ botânico fixado. As análises histoquímicas foram empregadas em material botânico in natura, seguindo-se protocolos específicos \\ para mucilagem, amido e sílica. Microanálises físicas foram feitas com Energy Dispersive Spectroscopy (EDS) detector. Nas \\ espécies estudadas de Oenocarpus Mart., as substâncias ergásticas foliares correspondem a mucilagem amorfa; grãos de amido \\ poliédricos do tipo simples e sílica opalina sob a forma de corpos elípticos e esféricos-globosos de superfície espiculada, \\ ambos com elevado teor de dióxido de silício. As observaçôes microscópicas, os testes histoquímicos e as microanálises físicas \\ permitiram diagnosticar, caracterizar e elucidar a estrutura e ultra-estrutura das substâncias ergásticas ocorrentes nas folhas \\ dos táxons analisados de Oenocarpus Mart.
}

PALAVRAS-CHAVE: Anatomia vegetal; metabólicos primários e secundários; histolocalização.

\section{Leaf ergastic substances of Amazonian species of Oenocarpus Mart. (Arecaceae): histochemical and ultrastructural characterization}

\section{ABSTRACT}

The purpose of this study was to diagnose and characterize the leaf ergastic substance of Oenocarpus bacaba Mart., O. distichus Mart., O. mapora H. Karst. and O. minor Mart. by light and scanning electron microscopy, histochemical analyses and physical microanalyses. Transverse and longitudinal sections as well as maceration were made in fixed botanical material. The histochemical analyses were made in non-fixed botanical material, using specific tests for mucilage, starch and silica. An Energy Dispersive Spectroscopy (EDS) detector was used for the physical microanalyses. In the Oenocarpus Mart. species studied, the leaf ergastic substances corresponded to amorphous mucilage, simple-type polyhedrical starch grain and opaline silica in the shape of elliptical and spherical, globular of pointed surface bodies, both with silicon dioxide in the correct concentration. The microscopic observations, histochemical analyses and physical microanalyses permitted us to diagnose, characterize and elucidate the structure and ultrastructure of the ergastic substances occurring in the leaves of the analyzed Oenocarpus Mart. taxa.

KEYWORDS: Plant anatomy; primary and secondary metabolics; histolocalization.

\footnotetext{
1 Parte da dissertação de mestrado em Botânica Tropical do primeiro autor.

2 Museu Paraense Emílio Goeldi, Coordenação de Pesquisa e Pós-Graduação, Avenida Perimetral, 1901, CEP 66077-830, Caixa Postal: 399 - Belém -PA -Brasil.

${ }^{3}$ Museu Paraense Emílio Goeldi, Coordenação de Botânica, Avenida Perimetral, 1901,CEP 66077-830, Caixa Postal: 399 - Belém -PA -Brasil.

* Autor para correspondência (rolfjß@hotmail.com).
} 


\section{INTRODUÇÃO}

A família Arecaceae, considerada uma das mais comuns entre as Liliopsidas, é a única incluída na ordem Arecales (APG II, 2003), apresentando distribuição predominantemente pantropical (Cronquist, 1981). Possui cerca 1.500 espécies em 200 gêneros endêmicos pertencentes a seis subfamílias, sendo que nas Américas está representada por 550 espécies e 67 gêneros (Henderson et al., 1995). No Brasil, Souza e Lorenzi (2005) consideram 40 gêneros e 200 espécies.

As palmeiras, denominação vernácula das Arecaceae, constituem um dos componentes mais característicos da paisagem amazônica (Kahn, 1988), que abriga aproximadamente $50 \%$ dos gêneros e $30 \%$ das espécies de palmeiras Neotropicas (Henderson et al., 1995). Kahn e De Granville (1992) assinalam que das seis subfamílias definidas por Dransfield e Uhl (1986) para as Arecaceae, cinco possuem representantes na Amazônia: Coryphoideae, Calamoideae, Ceroxyloideae, Arecoideae e Phytelephantoideae. Destas, Arecoideae é a que apresenta o maior número de gêneros na região (28 gêneros), sendo Astrocaryum G. F. W. Meyer, Bactris N. J. Jacquin ex Scopoli, Geonoma Willdenow e Oenocarpus Martius os mais diversificados, que juntos correspondem a mais de 50\% do total de espécies. Para Oenocarpus Martius, Balick (1986) cita nove espécies que estão distribuídas no norte da América do Sul, extendendo-se ao norte para a América Central e, ao sul, para o Brasil e Bolívia.

A anatomia vegetal fornece importantes contribuiçóes à elucidação de relações ecológicas, taxonômicas e filogenéticas das plantas vasculares, assim como para a fisiologia das mesmas (Silva e Potiguara, 2008). Neste sentido, Uhl e Dransfield (1987) enfatizam que estudos anatômicos são significativos para a classificação e avaliação das inter-relações e mudanças evolutivas das palmeiras, visto que estas se mostram variáveis tanto na estrutura interna como na externa. Mesmo assim, as pesquisas sobre Arecaceae na Amazônia têm enfocado principalmente os aspectos alimentar, medicinal e sócioeconômico, conforme verificado em trabalhos etnobotânicos de Balick (1984), Jardim e Stewart (1994), Jardim e Cunha (1998), Rocha e Silva (2005) e outros, havendo escassez de estudos histoquímicos e ultra-estruturais de metabólicos como o realizado por Kikuchi et al. (2007).

As substâncias ergásticas, ou seja, produtos do metabolismo vegetal primário ou secundário, possuem grande valor taxonômico e filogenético em vários grupos vegetais (Essig, 1979), nos quais a utilizaçáo da microscopia eletrônica de varredura possibilitou ampliar o conhecimento celular e refinar os detalhes estruturais (Grimstone, 1980).

Desta forma, o presente trabalho objetivou diagnosticar e caracterizar as substâncias ergásticas foliares de $O$. bacaba Mart., O. distichus Mart., O. mapora H. Karst. e O. minor Mart. por meio das microscopias óptica e eletrônica de varredura, de análises histoquímicas e microanálises físicas, visando contribuir com o conhecimento biológico dos táxons analisados e subsidiar trabalhos futuros sobre fisiologia, ecofisiologia e taxonomia de Oenocarpus Martius e demais Arecaceae.

\section{MATERIAL E MÉTODOS}

As espécies de Oenocarpus Mart. estudadas foram coletadas em duas áreas do município de Belém, estado do Pará: no Parque Zoobotânico do Museu Paraense Emílio Goeldi (MPEG) e na Área de Proteção Ecológica do Guamá (APEG). Os espécimes estão depositados no Herbário João Murça Pires (MG) pertencente ao MPEG, com as seguintes especificaçôes: O. minor Mart. (MG 178490), O. distichus Mart. (MG 178491), O. mapora H. Karst. (MG 178492) e O. bacaba Mart. (MG 178493).

Para a análise anatômica, foram utilizadas folhas maduras

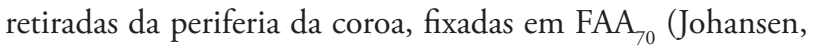
1940) por 24 horas e, posteriormente, conservadas em álcool a $70 \%$. Foram consideradas três regióes ao axis foliar (ráquis, pecíolo e bainha foliar) e pinas: apical, mediana e basal (Figura 1). Do axis foliar, foram retiradas amostras das regiōes periférica, central e mediana (Figura 2). Das pinas, foram selecionados o ápice, porção mediana e base do limbo, que foi subdividido em margem, nervura central e porção intermediária (Figura 3). Nestas amostras, foram realizadas maceraçấo e secçôes à mão livre. A maceração foi feita segundo Franklin (1945), mantendo-se as amostras em estufa a $55^{\circ} \mathrm{C}$ por duas semanas. Posteriormente, o macerado foi submetido à centrifugação em $4500 \mathrm{rpm}$ por 20 minutos, seguido de lavagem em água destilada. Sucessivas centrifugaçóes alternadas com lavagem em água destilada foram realizadas até o desaparecimento de traços do ácido, verificaçấo esta feita com o auxílio de fitas de pH. Para a coloração, foi utilizada safranina hidroalcoólica 5\% (Kraus e Arduin, 1997), montando-se o macerado em meio temporário segundo Purvis et al. (1964).

As secçóes à mão livre, transversais e longitudinais, foram feitas com auxílio de lâmina de barbear, clarificadas em solução aquosa de hipoclorito de sódio comercial na proporção de 2:1 $(\mathrm{v} / \mathrm{v})$, com posterior lavagem em água destilada, coloração segundo Johansen (1940) e montagem em glicerina 50\% aquosa entre lâmina e lamínula. Quando necessário, as amostras foram submetidas ao amolecimento de acordo com Carlquist (1982) e, após desidratação, infiltração e inclusão em parafina histológica segundo Johansen (1940), foram seccionadas em micrótomo rotativo na espessura de $20 \mu \mathrm{m}$, coradas segundo o mesmo autor e montadas em bálsamo-docanadá entre lâmina e lamínula.

Protocolos de Johansen (1940), Jensen (1962) e Salatino e Silva (1975) foram empregados em material botânico não 

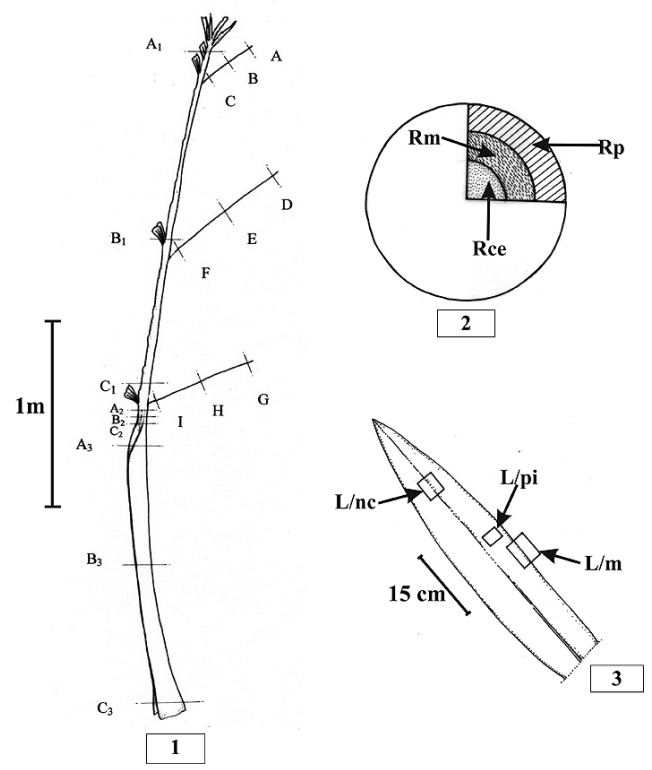

Figuras 1 a 3 - Representação esquemática das secções realizadas nas folhas das espécies estudadas de Oenocarpus Mart. 1: Representação geral. A: Ápice das pinas apicais; B: Porção mediana das pinas apicais; C: Base das pinas apicais; D: Ápice das pinas medianas; E: Porção mediana das pinas medianas; F: Base das pinas medianas; G: Ápice das pinas basais; H: Porção mediana das pinas basais; I: Base das pinas basais; $A_{1}$ : Ráquis apical; $B_{1}$ : Ráquis mediano; $\mathrm{C}_{1}$ : Ráquis basal; $\mathrm{A}_{2}$ : Pecíolo apical; $\mathrm{B}_{2}$ : Pecíolo mediano; $\mathrm{C}_{2}$ : Pecíolo basal; $\mathrm{A}_{3}$ : Bainha apical; $\mathrm{B}_{3}$ : Bainha mediana; $\mathrm{C}_{3}$ : Bainha basal. 2 : Axis foliar. Rce: Região central; Rm: Região mediana; Rp: Região periférica. 3: Pinas. L/mg: Limbo/margem; L/nc: Limbo/nervura central; L/pi: Limbo/ porção intermediaria.

fixado para o diagnóstico histoquímico de amido, sílica e mucilagem respectivamente.

Para o exame em microscopia eletrônica de varredura (MEV), amostras foliares desidratadas foram processadas em secador de ponto crítico de $\mathrm{CO}_{2}$, montadas em suportes metálicos e metalizadas com ouro com $20 \mathrm{~nm}$ de espessura por 150 segundos em corrente de $25 \mathrm{~mA}$.

Microanálises físicas para substâncias minerais foram realizadas com detector de Energy Dispersive Spectroscopy (EDS), acoplado ao MEV.

As fotomicrografias e eletromicrografias foram obtidas, respectivamente, em fotomicroscopio Zeiss e microscópio eletrônico de varredura Leo modelo $1450 \mathrm{VP}$, sendo as escalas micrométricas projetadas nas mesmas condiçôes ópticas.

\section{RESULTADOS E DISCUSSÃO}

As substâncias ergásticas foliares das espécies estudadas de Oenocarpus Mart. correspondem a sílica, mucilagem e amido.

A sílica, quimicamente um polímero amorfo de dióxido de silício hidratado $\left(\mathrm{SiO}_{2} \cdot \mathrm{nH}_{2} \mathrm{O}\right)$, ocorre sob a forma de incrustaçóes minerais opalinas chamadas de corpos silicosos por Tomlinson (1961). No interior das células epidérmicas das pinas e do axis foliar, a exceção da margem e bainha, os corpos silicosos mostram-se elípticos e cobertos por granulaçôes (Figuras 4 e 5). Corpos silicosos esféricos ocorrem associados a feixes fibrosos presentes no mesofilo das pinas e no tecido parenquimático do axis foliar, exceto na ráquis, estando localizados em stegmata que segundo Mettenius (1864) correspondem a idioblastos especializados na biossíntese e acúmulo deste tipo de incrustação mineral (Figuras 6 e 7). Em MEV, foi verificado que os stegmata dispõem-se em fileiras longitudinais, adjacentes e paralelas aos feixes de fibras, possuem paredes espessadas, forma discóide e uma cavidade ou depressão central na qual se situam os corpos de sílica, de aspecto esférico-globoso e superfície espiculada semelhantes a drusas (Figuras 8 e 9), tal como descrito para outras palmeiras (Vilhena et al., 1984; Rocha, 2004; Kikuchi et al., 2007). Microanálises realizadas por EDS revelaram que os corpos silicosos descritos acima apresentam elevado teor de dióxido de silício (Figura 10).
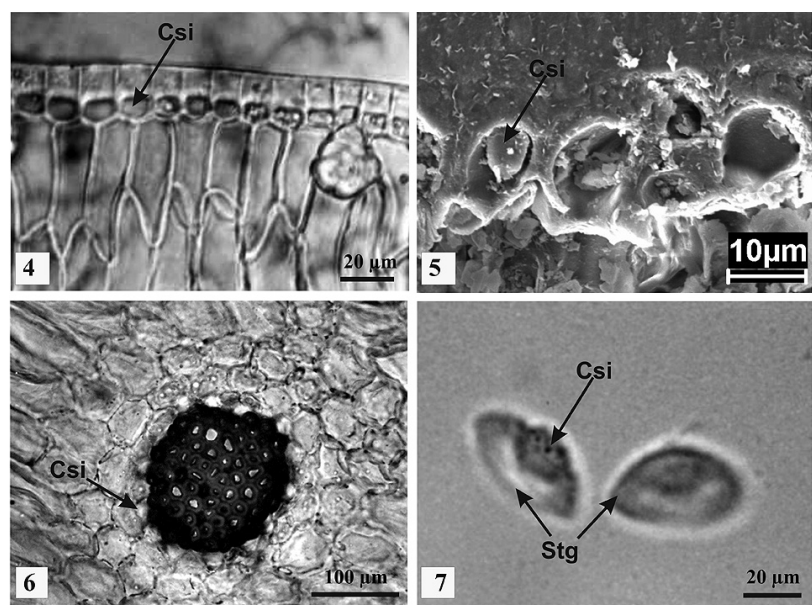

Figuras 4 a 7 - Substâncias ergásticas foliares de Oenocarpus Mart., sílica. 4 e 5: 0. bacaba Mart., evidenciando corpos silicosos elípticos. 4: Epiderme adaxial da pina em secção transversal; 5: Eletromicrografia de varredura da epiderme da região periférica da ráquis em seção transversal. 6 e 7: 0 . distichus Mart., evidenciando corpos silicosos esféricos. 6: Feixe fibroso da bainha foliar em secção transversal envolto por stegmata; 7: Macerado do pecíolo, detalhando corpos silicosos no interior de stegmata. Corpo silicoso (Csi); Stegmata (Stg).

A presença e a diversidade estrutural dos corpos de sílica são bem documentadas em diferentes famílias vegetais (Parthasarathy e Klotz, 1976; Simpson e Volcani, 1981) e citados como comuns as Arecaceae (Tomlinson, 1990) e outras Liliopsidas a exemplo das Bromeliaceae, Zingiberaceae, Orchidaceae, Pandanaceae e Cyclanthaceae (Dahlgren e Clifford, 1982). Contudo, questiona-se muito sobre os mecanismos fisiológicos que os formam, embora Haberlandt (1925) sugira que o ácido silícico absorvido pelas plantas do solo, por ser potencialmente tóxico as células, seja estabilizado 

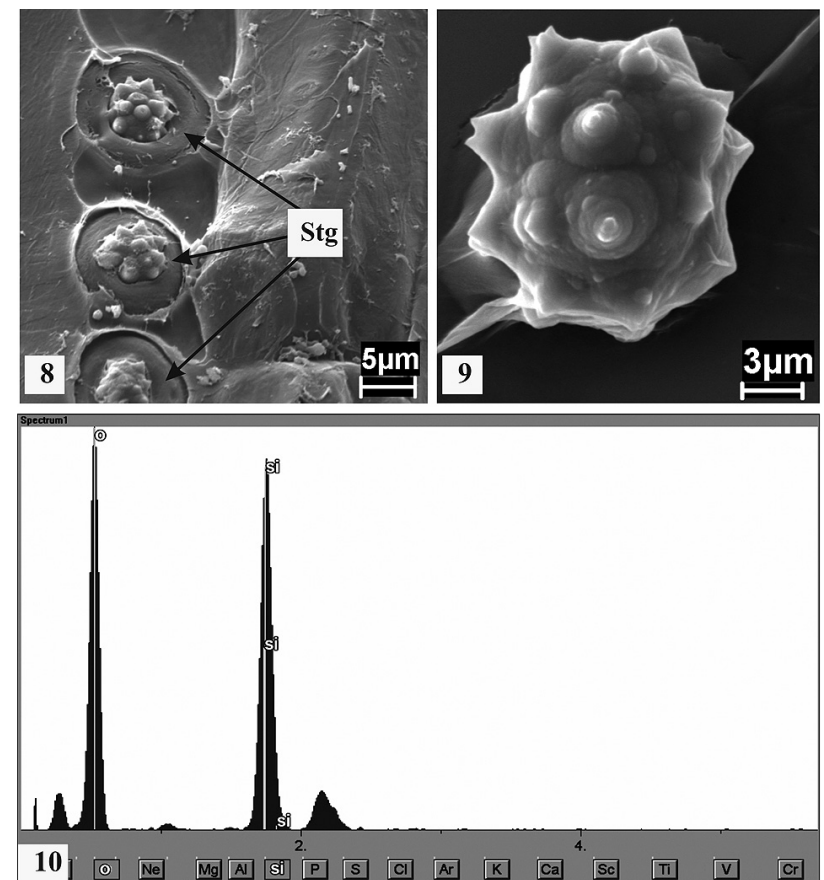

Figuras 8 a 10 - Substâncias ergásticas foliares de Oenocarpus Mart., sílica. 8 e 9: 0 . minor Mart., bainha foliar. 8: Eletromicrografia de varredura de um feixe fibroso evidenciando corpos silicosos esféricos-globosos de superfície espiculada no interior de stegmata; 9: Eletromicrografia de varredura detalhando um corpo silicoso esférico-globoso de superfície espiculada. 10: Espectro da análise por EDS realizada nos corpos silicosos, detectando elevado teor de dióxido de silício. Stegmata (Stg).

como biocomposto mineral e acumulado devido à ausência de um sistema excretor especializado.

Quanto à função, a sílica biogênica nas plantas tem sido associada à refletância das folhas (Campos e Labouriau, 1969), à proteção mecânica contra o ataque de insetos e patógenos (Paviani, 1972), ao controle da transpiraçấo excessiva e retenção de água (Sangster, 1977), à prevenção do colapso tecidual subjacente em condiçóes de seca (Metcalfe e Chalk, 1983), assim como ao balanço das trocas térmicas com o meio externo (Adatia e Besford, 1986). Em relação aos táxons aqui analisados de Oenocarpus Mart., acredita-se que a última e as duas primeiras funçôes sejam as mais prováveis, devido ao ambiente mesofítico onde os mesmos ocorrem.

Segundo Metcalfe e Chalk (1983), os corpos silicosos caracterizam espécies, gêneros e famílias de Liliopsidas, pois a ocorrência e morfologia destas incrustaçóes minerais são geneticamente determinadas e pouco influenciadas por fatores ambientais (Moller e Rassmussen, 1984). Sendo assim, a presença ou ausência deste tipo de inclusão celular representa um valor sistemático relevante para Liliopsidas como as Arecaceae (Prychid et al., 2004), na qual os corpos silicosos constituem uma condição plesiomórfica ao grupo (Chase et al., 2000), como também observado por Prychid et al. (2003) ao clado Zingiberales, Commelinales e Hanguana.

A mucilagem foi diagnosticada em estruturas secretoras internas do mesofilo e como impregnaçôes nas fibras perivasculares e não vasculares (Figuras 11 e 12). O macerado e a MEV mostraram, ainda, que esta substância faz-se presente no interior dos elementos de vaso sob a forma de corpos mucilaginosos, provavelmente resultantes do efeito coagulante exercido pelos solventes utilizados na preparaçáo das amostras (Figuras 13 e 14).

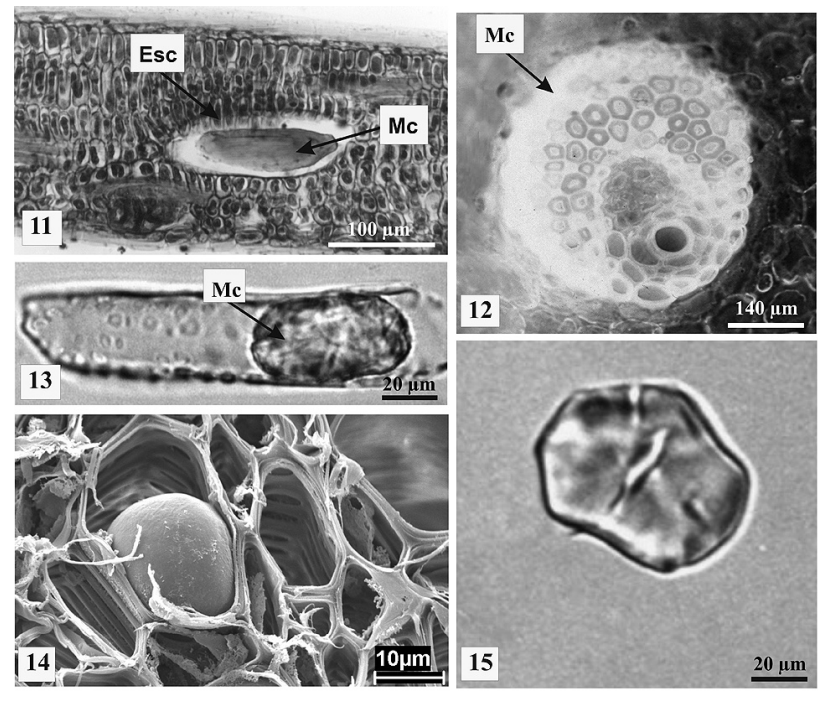

Figuras 11 a 15 - Substâncias ergásticas foliares de Oenocarpus Mart., mucilagem e amido. 11 e 12: 0. minor Mart. 11: Secção longitudinal do mesofilo evidenciando mucilagem no interior da estrutura secretora; 12 : Feixe vascular da ráquis evidenciando bainha fibrosa impregnada por mucilagem em luz polarizada. 13 e 14: O. mapora H. Karst. 13: Corpo mucilaginoso no interior do elemento de vaso da ráquis; 14 : Eletromicrografia de varredura de um feixe vascular do pecíolo, evidenciando corpo mucilaginoso no interior do vaso. 15: 0. distichus Mart., evidenciando grão de amido da ráquis. Estrutura secretora (Esc); Mucilagem (Mc).

Do ponto de vista químico, Costa (1994) considera que mucilagens correspondem a substâncias macromoleculares de natureza glicídica (polissacarídeos ácidos ou neutros) e com propriedades hidrofílicas. Devido a estas características, Eames e MacDaniels (1925) e Fahn (1979) atribuem-lhe diversas funçóes na economia da planta tais como: substância de reserva alimentar, aumento da capacidade de retenção de água e contra a perda da mesma nas células. As duas últimas funçóes sugerem que as mucilagens contribuem para a adaptaçáo das plantas a ambientes xéricos, conforme verificado por Rocha et al. (2002) nas malváceas Hibiscus tiliaceus L. e H. pernambucensis Arruda.

Como as espécies de Oenocarpus Mart. aqui estudadas foram coletadas em ambiente com características mesofíticas, infere-se que a mucilagem verificada nas folhas não corresponda a uma adaptaçáo ecofisiológica e sim a um 
caractere químico inerente ao genótipo dos táxons. Ademais, as propriedades físico-químicas das mucilagens explicam sua presença no interior dos elementos de vaso, uma vez que se solubilizam em água e, por conseguinte, difundem-se pelos tecidos e impregnam estruturas consoante descrito.

Grãos de amido simples de contornos poliédricos, com faces ligeiramente côncava e convexa, hilo fendido e cêntrico e sem zonas concêntricas aparentes foram visualizados apenas no macerado do axis foliar das espécies (Figura 15).

Salisbury e Ross (1992) comentam que o amido corresponde a um heteropolímero polissacarídico de reserva resultante da condensação de dois polímeros de glucose: um essencialmente linear, denominado amilose, e outro bastante ramificado, chamado amilopectina, cuja forma varia de esférica a elíptica. Para Bewley e Black (1994), a geometria dos grãos de amido é determinada principalmente pelo conteúdo de amilose, já que se tornam mais esféricos à medida que ocorre incremento da amilose em relação a amilopectina. Assim, supóe-se que os grãos amilíferos dos táxons estudados de Oenocarpus Mart., para o estágio de desenvolvimento em que foram coletados, possuam quantidades superiores de amilose e, por esta razão, assumem formato poliédrico tendendo a circular, pois segundo Cereda e Franco (2002) os grânulos amilíferos apresentam em geral estrutura e propriedades funcionais modificadas durante a ontogenia da planta, como constatado por Amaral et al. (2001) nas sementes de Bixa orellana $\mathrm{L}$.

De acordo com Meyer et al. (1965) e Costa (1994), os grãos de amido embora variem em tamanho e número apresentam morfologia praticamente constante para uma determinada espécie, sendo por isso utilizados nas identificaçôes taxonômicas (Galliard, 1987) e arqueobotânicas (Freitas, 2002). Contudo, Tomlinson (1961) assinala que não existe diversidade morfológica de grãos amilíferos entre as palmeiras para os tornarem úteis como caractere diagnóstico. A afirmação do último autor está em acordo com as constatações feitas no presente estudo, visto que os gráos de amido descritos sấo comuns aos táxons estudados de Oenocarpus Mart.

As observaçôes microscópicas, os testes histoquímicos e as microanálises físicas permitiram não apenas diagnosticar a natureza das substâncias ergásticas foliares das espécies de Oenocarpus Mart. aqui analisadas, como também elucidar a ultra-estrutura das mesmas em especial pelo uso do microscópio eletrônico de varredura. Ressalta-se, todavia, a necessidade da ampliação de estudos que versem sobre metabólicos nos demais representantes de Oenocarpus Mart. e das Arecaceae como um todo, visando ratificar aqueles que foram observados e detectar outros úteis as interpretaçôes fisiológicas, ecofisiológicas, taxonômicas e filogenéticas.

\section{AGRADECIMENTOS}

Ao Conselho Nacional de Desenvolvimento Científico e Tecnológico $(\mathrm{CNPq})$, pela concessão da bolsa de Mestrado. Ao ilustrador científico Carlos Alvarez, pelos desenhos em planta baixa.

\section{BIBLIOGRAFIA CITADA}

Adatia, M.H.; Besford, R.T. 1986. The effects of silicon on Cucumber plants grown in recirculating nutrient solution. Annals of Botany, 58: 343-351.

Amaral, L.I.V. do; Pereira, M. de F.D.A.; Cortelazzo, A.L. 2001. Formação das substâncias de reserva durante o desenvolvimento de sementes de urucum (Bixa orellana L. - Bixaceae). Acta Botanica Brasilica, 15(1): 125-132.

Angiosperm Phylogeny Group. 2003. An update of the Angiosperm Phylogeny Group classification for the orders and families of flowering plants: APG II. Botanical Journal of the Linnean Society, 141: 399-436.

Balick, M. 1984. Ethnobotany of palms in the neotropics. Advances in Economic Botany, 1: 9-23.

Balick, M. 1986. Systematics and economic botany of the Oenocarpus-Jessenia (Palmae) complex. Advances in Economic Botany, 3: 1-140.

Bewley, J.D.; Black, M. 1994. Seeds: physiology of development and germination. 2. ed. Plenum Press, New York. 445pp.

Campos, A.C.; Labouriau, L.G. 1969. Corpos silicosos de gramíneas dos cerrados II. Pesquisa Agropecuária Brasileira, 4: 143-151.

Carlquist, S. 1982. The use of ethylenediamine in softening hard plant structures for paraffin sectioning. Stain technology, 57(5): 311-317.

Cereda, M.P.; Franco, C.L. 2002. Propriedades gerais do amido. Fundação Cargill, São Paulo. 221pp.

Chase, M.W.; Soltis, D.E.; Soltis, P.S.; Rudall, P.J.; Fay, M.E.; Hahn, W.H.; Sullivan, S.; Joseph, J.; Molvray, M.; Kores, P.J.; Givnish, T.; Sytsma, K.J.; Pires, C. 2000. Higher-level systematics of the monocotyledons: an assessment of current knowledge and a new classification. In: Wilson, K.L.; Morrison, D.A. (Eds). Monocots: systematics and evolution. Vol. 1. CSIRO, Melbourne, Australia. p. 3-16.

Costa, A.F. 1994. Farmacognosia. 4. ed. Fundação Calouste Gulbenkian, Lisboa. p. 1-91.

Cronquist, A. 1981. An integrated system of the classification of flowering plants. Columbia University Press, New York. p. 1082-1086.

Dahlgren, R.M.T.; Clifford, H.T. 1982. The monocotyledons: a comparative study. Academic Press, London. p. 38-90.

Dransfield, J.; Uhl, N.W. 1986. An outline of the classification of palms. Principes, 30(1): 3-11.

Eames, A.J.; MacDaniels, L.H. 1925. An introduction to plant anatomy. McGraw-Hill, New York. 427pp.

Essig, F. 1979. Trends of specialization in the palm pericarp. In: Henderson, A.; Borchsenius, F. (Eds.). Evolution, variation and classification of palms. Botanical Garden, New York. p. 73-77. 
Fahn, A. 1979. Secretory tissues in plants. Academic Press, London. p. 115-128.

Franklin, G.L. 1945. Preparation of thin sections of synthetic resins and wood-resin composites, and a new macerating method for wood. Nature, 51(1): 39-24.

Freitas, F. de. 2002. O uso de grãos de amido na identificação e análise de materiais arqueológicos vegetais. Brasília: Embrapa Recursos Genéticos e Biotecnologia. Boletim de Pesquisa e Desenvolvimento, n. 23,26 p.

Galliard, T. 1987. Starch availably and utilization. In: Galliard, T. (Ed.). Starch: proprieties and potential. John Wiley and Son, Brisbane. p. 1-15.

Grimstone, A.V. 1980. O microscópio eletrônico em biologia. EPU-São Paulo, São Paulo. 70pp.

Haberlandt, G. 1925. Physiological plant anatomy. Today \& tomorrow's, Delhi. p. 529-613.

Henderson, A.; Galeano, G.; Bernal, R. 1995. Field guide to the Palms of the Americas. Princeton University Press, New Jersey. 418pp.

Jardim, M.A.G.; Cunha, A.C.C. 1998. Usos de palmeiras em uma comunidade ribeirinha do estuário amazônico. Boletim do Museu Paraense Emílio Goeldi, série Botânica, 14(1): 33-41.

Jardim, M.A.G.; Stewart, P.J. 1994. Aspectos etnobotânicos e ecológicos de palmeiras no município de Novo Airão, Estado do Amazonas, Brasil. Boletim do Museu Paraense Emílio Goeldi, série Botânica, 10(1): 69-76.

Jensen, W.A. 1962. Botanical histochemistry: principles and practice. W.H. Freeman, San Francisco. 408pp.

Johansen, D.A. 1940. Plant microtechnique. MacGraw-Hill Book Co, New York. 523pp.

Kahn, F. 1988. Species richness and density of palms in terra firme forest of Amazonia. Biotropica, 20(4): 266-269.

Kahn, F.; De Granville, J.J. 1992. Palms in forest ecosystems of Amazonia. Ecological Studies 95. Springer-Verlag, Berlin. 226pp.

Kikuchi, T.Y.P.; Potiguara, R.C. de V.; Santos, P.P. dos. 2007. Caracterização histoquímica e ultra-estrutural do estipe de Socratea exorrhiza (Mart.) H. Wendl. (Arecaceae). Boletim do Museu Paraense Emílio Goeldi, Ciências Naturais, 2(2): 61-68.

Kraus, J.E.; Arduin, M. 1997. Manual básico de métodos em morfologia vegetal. EDUR, Rio de Janeiro. 198pp.

Metcalfe, C.R.; Chalk, L. 1983. Anatomy of the dicotyledons-Wood structure and conclusion of the general introduction. 2. ed. Claredon Press, Oxford. 308pp.

Mettenius, G.H. 1864. Über die Hymenophyllaceae. Abhandlungen der Königlich-Sächsischen Gesellschaft der Wissenschaften Mathematisch-Physikalischen Classe, 7: 403-504.

Meyer, B.S.; Anderson, D.B.; Böhning. 1965. Introdução à fisiologia vegetal. Fundação Calouste Gulbenkian, Lisboa. p. 257.

Moller, J.D.; Rasmussen, F.L.S. 1984. Stegmata in Orchidales: character state distribution and polarity. Botanical Journal the Linnean Society, 89: 53-76.

Parthasarathy, M.V.; Klotz, L.H. 1976. Palm "wood” I: Anatomical aspects. Wood Science Technology, 10: 215-229.
Paviani, T.I. 1972. Estudos morfológico e anatômico de Brasilia sickii G.M. Barroso: I. Revista Brasileira de Biologia, 32(4): 451-472.

Prychid, C.J.; Furness, C.A.; Rudall, P.J. 2003. Systematic significance of cell inclusions in Haemodoraceae and allied families: silica bodies and tapetal raphides. Annals of Botany, 92: 571-580.

Prychid, C.J.; Rudall, P.J.; Gregory, M. 2004. Systematics and biology of silica bodies in monocotyledons. Botanical Review, 69: 377-440.

Purvis, M.J.; Collier, D.C.; Walls, D. 1964. Laboratory techniques in botany. Butterwoths, London. p. 152.

Rocha, A.E.S. da; Silva, M.F.F. da. 2005. Aspectos fitossociológicos, florísticos e etnobotânicos das palmeiras (Arecaceae) de floresta secundária no município de Bragança, PA, Brasil. Acta Botanica Brasilica, 19(3): 657-667.

Rocha, C.B.R. 2004. Anatomia das folhas e análise quantitativa das fibras de Astrocaryum murumuru var. murumuru Mart. (Arecaceae). Dissertação de Mestrado, Universidade Federal Rural da Amazônia/Museu Paraense Emílio Goeldi, Belém, Pará. 57pp.

Rocha, J.F.; Neves, L. de J.; Pace, L.B. 2002. Estruturas secretoras em folhas de Hibiscus tiliaceus L. e Hibiscus pernambucensis Arruda. Revista da Universidade Rural do Rio de Janeiro, série Ciências da Vida, 22(1): 43-55.

Salatino, A.; Silva, J.B. 1975. Anatomia e óleos essências das folhas de Piper regnellii (Miq.) C.D.C var. regnellii. Boletim de Botânica da Universidade de São Paulo, 3: 95-106.

Salisbury, F.B.; Ross, C.W. 1992. Plant physiology. 4. ed. Wadsworth, Belmont. p. 267.

Sangster, A.G. 1977. Characteristics of the silica deposition in Digitaria sanguinalis (L.) Scop. (Crabgrass). Annals of Botany, 41: 341-350.

Silva, R.J.F.; Potiguara, R.C. de V. 2008. Aplicaçốes taxonômicas da anatomia foliar de espécies amazônicas de Oenocarpus Mart. (Arecaceae). Acta Botanica Brasilica, 22(4): 999-1014.

Simpson, T.L.; Volcani, B.E. 1981. Silicon and siliceous structure in biological systems. Springer-Verlag, New York. p. 495-525.

Souza, V.C.; Lorenzi, H. 2005. Botânica Sistemática. Instituto Plantarum, São Paulo. 640pp.

Tomlinson, P.B. 1961. Anatomy of the monocotyledons-II: Palmae. Oxford University Press, New York. 462pp.

Tomlinson, P.B. 1990. The structural biology of palms. Oxford University Press, New York. 460pp.

Uhl, N.W.; Dransfield, J. 1987. Genera Palmarum: a classification of palms based on the work of Harold E. Moore Jr. Allen Press, Kansas. 610pp.

Vilhena, R.C.Q. de; Lins, A.; Luna, M.S. 1984. Estudo anatômico, morfológico e do crescimento das raízes adventícias de Iriartea exorrhiza Mart. (Palmae), "Paxiúba". Boletim do Museu Paraense Emílio Goeldi, série Botânica, 1(1/2): 87-109.

Recebido em 12/08/2008

Aceito em 02/08/2009 